\title{
Progress on the Development of a Wavelength-shifting Optical Module
}

\author{
Dustin Hebecker ${ }^{\star a}$, Markus Archinger ${ }^{c}$, Sebastian Böser ${ }^{c}$, Esther Del Pino \\ Rosendo $^{c}$, Michael DuVernois ${ }^{d}$, Peter Falke $^{e}$, Carl-Christian Fösig $^{c}$, Timo Karg $^{b}$, \\ Lutz Köpke $^{c}$, Marek Kowalski ${ }^{a b}$, Krystina Sand ${ }^{c}$ and Delia Tosi ${ }^{d}$ \\ ${ }^{a}$ Institut für Physik, Humboldt-Universität zu Berlin \\ D-12489 Berlin, Germany \\ ${ }^{b} D E S Y$ \\ D-15738 Zeuthen, Germany \\ ${ }^{c}$ Institute of Physics, University of Mainz \\ Staudinger Weg 7, D-55099 Mainz, Germany \\ ${ }^{d}$ Dept. of Physics and Wisconsin IceCube Particle Astrophysics Center \\ University of Wisconsin, Madison, WI 53706, USA \\ ${ }^{e}$ Physikalisches Institut, Universität Bonn \\ Nussallee 12, D-53115 Bonn, Germany \\ E-mail: dustin.hebeckeredesy.de
}

\begin{abstract}
We report on the development of a single-photon sensor that employs wavelength-shifting and light-guiding techniques to maximize the collection area and to minimize the dark noise rate. The sensor is tailored towards application in ice-Cherenkov neutrino detectors using inert and cold, low-radioactivity and UV transparent ice as a detection medium, such as IceCube-Gen2 or MICA. The goal is to decrease the energy threshold as well as to increase the energy resolution and the vetoing capability of the neutrino telescope, when compared to a setup with optical sensors similar to those used in IceCube. The proposed sensor captures photons with wavelengths between $250 \mathrm{~nm}$ and $400 \mathrm{~nm}$. These photons are re-emitted with wavelengths above $400 \mathrm{~nm}$ by a wavelength shifter coating applied to a $90 \mathrm{~mm}$ diameter polymer tube which guides the light towards a small-diameter PMT via total internal reflection. By scaling the results from smaller laboratory prototypes, the total efficiency of the proposed detector for a Cherenkov spectrum is estimated to exceed that of a standard IceCube optical module by a factor of $\approx 2$. The status of the prototype development and the performance of its main components will be discussed.
\end{abstract}

The 34th International Cosmic Ray Conference,

30 July- 6 August, 2015

The Hague, The Netherlands

\footnotetext{
* Speaker.
} 


\section{Motivation}

To detect low photon rates in current large-scale scintillation and water Cherenkov detectors large area photomultiplier tubes (PMTs) or multiple smaller PMTs are employed. To first order, the energy threshold is determined by the photocathode area per unit volume. Furthermore the noise is directly proportional to the PMTs photosensitive area. With higher noise rates a lowenergy event can be mimicked by a random coincidence of noise hits which thus directly affect the detector's energy threshold. We present here an optical module that is supposed to lower these bounds and improve on all these parameters. The Wavelength-shifting Optical Module (WOM) is a low cost single-photon detector, with an increased photosensitive area and a reduced overall noise. This is achieved by adding a wavelength-shifting and a light-guiding tube as an additional passive component to the PMT. The wavelength shifter (WLS) shifts light from the UV (250-400 nm) towards the blue $(>400 \mathrm{~nm})$. As the amount of Cherenkov photons is proportional to $\frac{1}{\lambda^{2}}$ and most scintillators emit in the UV it is beneficial to be most sensitive in the UV. The WLS is a mean to work around Liouville's theorem and capture light in the tube, it also allows to detect photons with wavelengths that are originally too short to be detected by a PMT. The detection of shifted photons in the WOM is done by smaller and less noisy PMTs at the end of the tube.

In large volume detectors like IceCube-Gen2 [1], this allows the energy threshold to be lowered while keeping the detector volume and amount of deployed modules constant. In addition the high sensitive area to noise ratio allows for good vetoing capabilities. These properties are often desired and make this concept interesting not only for large volume neutrino experiments [1, 2], but also for experiments like SHiP [3].

\section{Introduction to the Concept}

The WOM as shown in Fig. 1 consists of a pressure vessel, a wavelength-shifting and light guiding tube, an adiabatic light guide and PMTs with their readout electronics. The pressure vessel protects the sensitive components inside from the pressure and adds a small air layer around the the light guide to enhance its light-guiding capabilities. It is $1.3 \mathrm{~m}$ long and $114 \mathrm{~mm}$ in diameter and made from quartz glass in order to be transparent to the UV-range of the Cherenkov spectrum. The wavelength-shifting and light guiding tube within the pressure vessel is a $90 \mathrm{~mm}$ diameter tube made of PMMA. ${ }^{1}$. The tube is coated with a custom made wavelength-shifting paint [4]. UV-light interacting with the WLS is absorbed and isotropically re-emitted at a lower wavelength. The air gap between pressure vessel and inner tube allows the isotropically emitted light to be captured by total internal reflection (theoretically $74.6 \%$ ). The captured light travels in the tube to the upper or lower end where an adiabatic light guide is glued to the tube. The adiabatic light guide virtually losslessly directs the light from the tube walls towards a PMT at its end. Several low noise PMTs are considered for the readout, such as the Hamamatsu R11920-100. The readout electronics will be an adapted version of the Gen2 Digital Optical Module (Gen2-DOM) [5] electronics. In the following sections we will give a short overview of the custom made WLS paint we developed and its properties when coated on small $(30 \mathrm{~cm}$ long and $2 \mathrm{~cm}$ diameter) PMMA test tubes. Also we will present preliminary noise measurements obtained with the R11920-100 PMT. We will

\footnotetext{
${ }^{1}$ Acrylic glass
} 


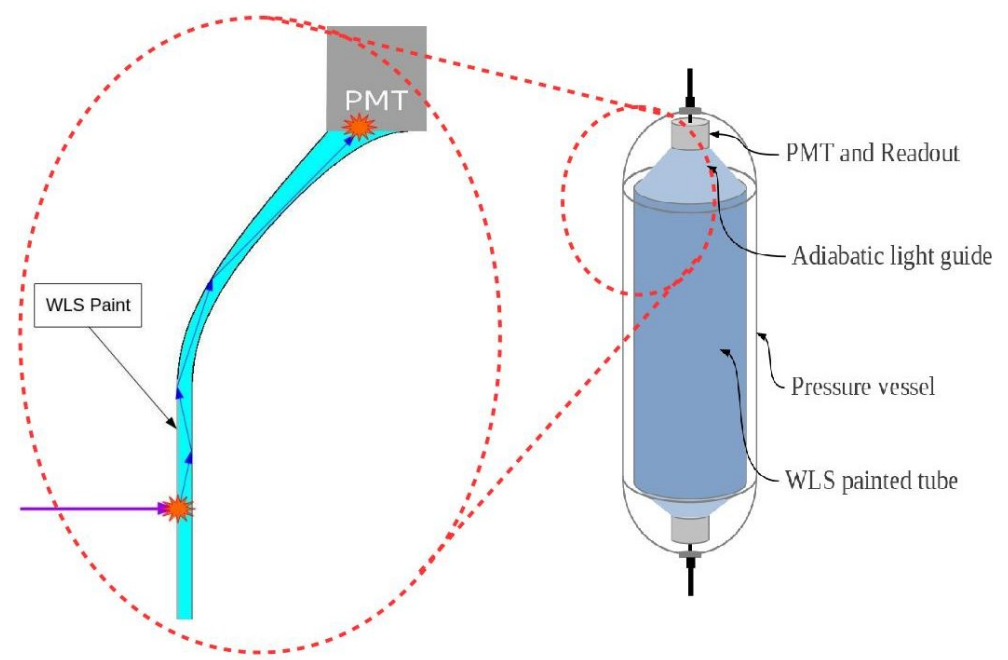

Figure 1: Schematic of the wavelength-shifting Optical Module (WOM) on the right. The working principle of a wavelength-shifting light guide is shown in a cross section on the left. Light is absorbed by a thin layer of WLS and re-emitted isotropically at a lower wavelength. With a probability of $\approx 75 \%$ the re-emitted light is captured by total internal reflection and guided towards the PMT where it can be detected.

show our progress with the electronics as well as present our steps towards assembling a functional prototype.

\section{Efficiency}

We have put extensive investigation in developing the WLS paint and understanding its properties when coated on a transparent light guiding tube. The key value for this is the capture and transport efficiency $\varepsilon$. In the following $\varepsilon$ is defined as the ratio of shifted photons leaving on either side of a tube coated with a WLS paint to the amount of UV photons the tube is exposed to. This value has been obtained in different ways. They all have in common that the test tube is illuminated with a small light beam perpendicular to the tube axis and the light coming out at one end is detected. The other end is blackened to reduce reflections. The efficiency $\varepsilon$ was used to choose and develop the most efficient paint, for selection of the right substrate and for estimating the final detector efficiency.

\subsection{WLS Paint Development}

First measurements were done with EJ-298 [6], a commercial WLS paint. EJ-298 showed a peak efficiency $\varepsilon$ close to $23 \%$ for a narrow wavelength range. After several tests with custom made WLS paints were made to improve the efficiency $\varepsilon$, similar efficiencies but over a broader absorption range where obtained. The solvents, binders and fluorescent dyes investigated are shown in Tab. 1 . The paint performing best in efficiency $\varepsilon$, adhesiveness and surface quality ${ }^{2}$, was made with

\footnotetext{
${ }^{2}$ Evaluated by visual inspection
} 

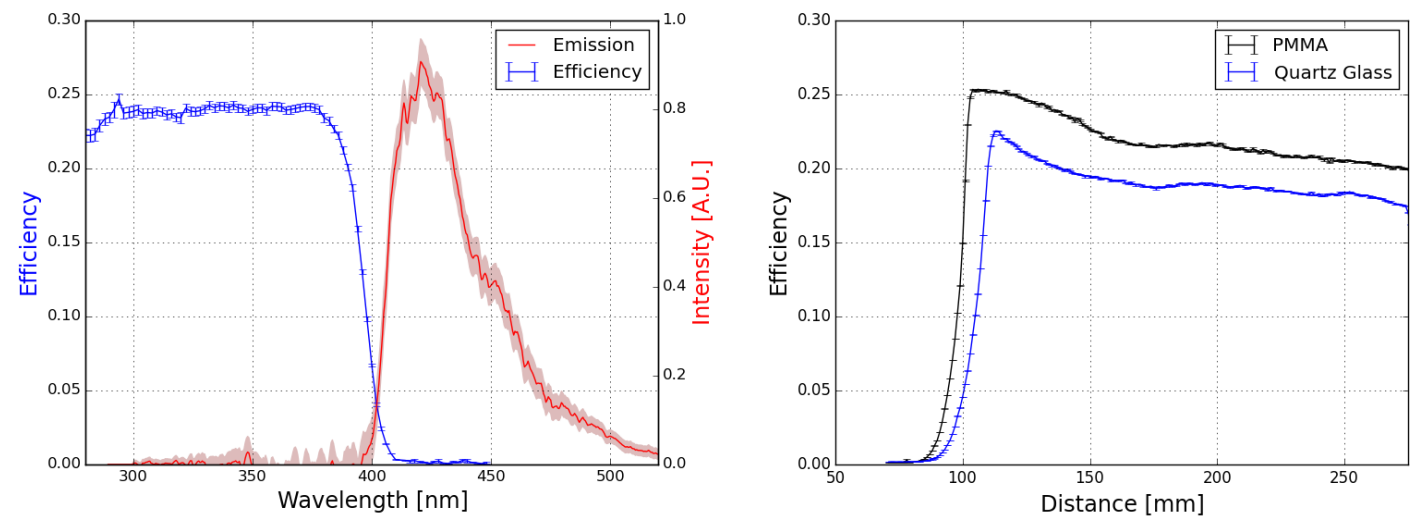

Figure 2: Efficiency $\varepsilon$ of a custom made WLS paint coated on a PMMA tube for light capturing and transport measured with a photodiode and the emission spectrum of the same paint (left).Efficiency $\varepsilon$ measured as a distance between detecting photodiode and light source along the test tube for $360 \mathrm{~nm}$. The absolute efficiency $\varepsilon$ and decay behavior for quartz glass and PMMA are shown in comparison. The efficiency $\varepsilon$ falls off below $100 \mathrm{~mm}$ because there is no WLS coating on the tube beyond this distance. Both functions are corrected for geometric efficiency (right).

\begin{tabular}{|l|l|}
\hline Binder & WLS \\
\hline DIAKON MG102D (PMMA) & Butyl-PBD (2-(4-Biphenylyl)-5-(4-t-butylphenyl)-1,3,4-oxadiazol) \\
Paraloid B67 & PQP (p-Quarterphenyl) \\
Paraloid B44 & TPB (1,1,4,4-Tetrapenyl-1,3-butadiene) \\
Paraloid B72 & QUI (3,5,3"",5"'-Tetra-t-butyl-p-quinquephenyl) \\
Paraloid B82 (PMMA) & Bis-MSB (p-Bis(o-methylstyryl)-benzene) \\
\hline Solvent & WLS \\
\hline Toluene & P-Therphenyl \\
P-Xylene & TMI (2,5,2"",5"'-Tetramethyl-p-quinquephenyl) \\
Dichloromethane & PBD (2-(4-Biphenylyl)-5-phenyl-1,3,4-oxadiazol) \\
Anisole & BBD (2,5-Bis-(4-biphenylyl)-1,3,4-oxadiazol) \\
& PMP (1-phenyl-e-mesityl-2-pyrazoline) \\
\hline
\end{tabular}

Table 1: Binders, solvents and WLS dyes investigated during the development of a WLS paint development.

a mixture of $77.31 \%^{3}$ Toluene, $22.29 \%$ Paraloid B72 ${ }^{4}, 0.13 \%$ Bis-MSB and $0.27 \%$ P-Terphenyl [4]. In order to dissolve the plastic and the WLS dyes in the solvent, the solvent was heated just below the boiling point. In order to avoid clogging, the plastic and the WLS dyes were added in small additional dosages after each previous dosage had been dissolved. The efficiency and emission spectra of this paint are shown in Fig. 2 (left) ${ }^{5}$. To apply the paint to the substrate a dip-coating process is used, where the substrate is immersed in WLS paint and then withdrawn with a well defined velocity. The higher the withdraw velocity the thicker the paint film on the substrate. With the

\footnotetext{
${ }^{3}$ Percentage by weight.

${ }^{4}$ ethyl-methacrylate copolymer

${ }^{5}$ The attentive reader may notice a difference in efficiency $\varepsilon$ to the 2013 ICRC contribution [7]. The then rather high efficiency $\varepsilon$ has been caused by a software bug.
} 
above mentioned mixture a velocity of $9.3 \mathrm{~cm} / \mathrm{min}$ has given the best balance between absorption and surface quality.

\subsection{Substrate}

The behaviour of different substrates for the paints was investigated as well. The efficiency $\varepsilon$ as function of the light source position along the test tube is shown in Fig. 2 (right) where quartz glass and PMMA are compared. PMMA was chosen as substrate due to its better performance and adhesive properties in combination with most of the paints. The decay in efficiency $\varepsilon$ in Fig. 2 (right) indicates a "loss length" along the tube of $\approx 0.8 \mathrm{~m}$ if exponential behavior is assumed. Note that scattering or attenuation length may be different to the loss length as the light will travel further on a spiral path. To increase the loss length cleaner materials will be explored. Also a reduction of vibrations in the dip-coating process, may increases the surface quality and thus the loss length. To limit the propagation losses, WLS tubes of $90 \mathrm{~cm}$ length are used for now.

\subsection{Theoretical Expectations}

The efficiency $\varepsilon$ to this point has been measured with a photodiode setup as described in [7]. The measured values are lower than our expectations considering a geometrical capture efficiency of $74.6 \%$ and the attenuation estimated from Fig. 2.

Several reasons for the observed efficiency are under study. With a modified setup we measured a quantum efficiency of $>90 \%$ for the WLS itself. This was done by illuminating a WLS paint coated glass surface with UV-light and capturing the emission at an angle that ensured no direct reflection of non-shifted UV-light. After confirming that the angular distribution of the emission is consistent with a Lambertian emitter we compared the measured emission to the reflection of a Speactralon [8] plate, a Lambertian scatterer used as reference. The result is consistent with the quantum efficiency quoted by the manufacturer of the commercial paint for their product.

A measurement of the photodiode's angular acceptance showed that part of the light under large incoming angles was not detected. For this measurement we used a cylindrical lens attached to the photodiode by optical grease. Then this setup was illuminated with parallel light under different angles. The angular distribution of the light from the tube is not known. Therefore the amount of unaccounted in-efficiency, due to reduced angular acceptance, can not be estimated yet.

As the final detector foresees the use of PMTs, we are currently investigating the test tubes with a PMT setup that has complementary instrumental uncertainties. First results indicate that the efficiency $\varepsilon$ might be significantly higher than previously thought.

\section{Electronics and Noise}

Besides increasing the effective area and improving the UV sensitivity, the WOM is designed to achieve low noise rates. For initial tests, the 8 dynode R11920-100 PMT from Hamamatsu (introduced by the CTA collaboration [9]) was chosen. This PMT has a 1.5" diameter, a peak quantum efficiency of $40 \%$ and a collection efficiency higher than $94 \%$.

To investigate the PMT performance at low temperatures, noise rate measurements where taken with the PMT in a dark box inside a climate chamber. For the readout, commercial 5 Gs/s FADC, $0.5 \mathrm{Gs} / \mathrm{s}$ FADC and a $1 \mathrm{GHz}$ oscilloscope were explored as well as a $0.25 \mathrm{Gs} / \mathrm{s}$ prototype board 
developed for the IceCube-Gen2 optical modules. The latter is an ALTERA development board including a Cyclone V FPGA [10]. For the short PMT pulses the development board setup needs to be modified to overcome its insufficient bandwidth.
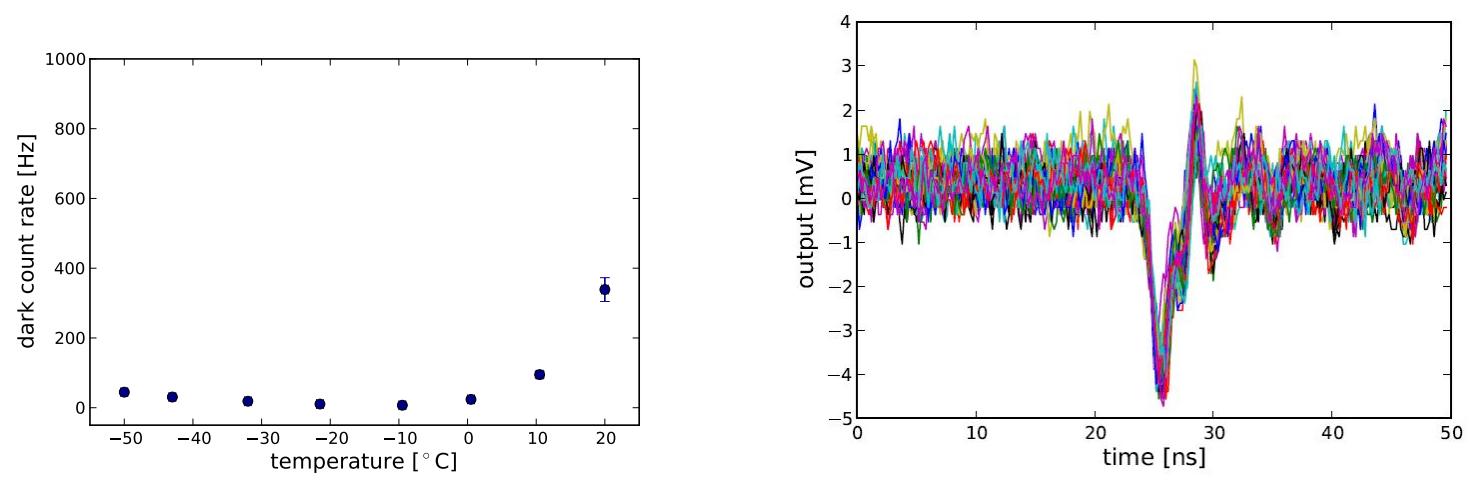

Figure 3: Dark count rates assuming a Gaussian SPE distribution as function of temperature for the R11920 PMT (left). Waveform with peak height in the range of single PE-peak at $-50^{\circ}$ (right).

The photocathode temperatures in IceCube-Gen2 range between $\sim-5^{\circ} \mathrm{C}$ at $2625 \mathrm{~m}$ and $\sim$ $-33^{\circ} \mathrm{C}$ at $1375 \mathrm{~m}$ depth corresponding to PMT dark count rates between 11 and $19 \mathrm{~Hz}$. Noise rates were measured with the oscilloscope and cover this range as shown in Fig. 3 (left). The counts were determined by fitting a Gaussian to the high side of the single photo-electron peak and then taking the total area. Typical waveforms are depicted in Fig. 3 (right). To measure the noise rate of the full WOM, the setup was assembled as shown in Fig. 4. While first tests were conducted, more data needs to be taken to quote numbers. For future measurements, we are looking into higher gain PMTs or suitable preamplifiers as well as customized readout systems.

\section{Outlook - Prototyping}

\subsection{Outer Pressure Vessel}

The quartz glass pressure housing has undergone static pressure testing up to 5250 psi (362 bar). After the test some optical defects were seen in the glass, but they may have been present prior to the testing. Repeated tests did not result in additional, or modified, glass features. No cracking was observed. Feedthrough connectors of the Seacon type previously employed in IceCube DOMs can be mounted on the quartz glass housing as needed.

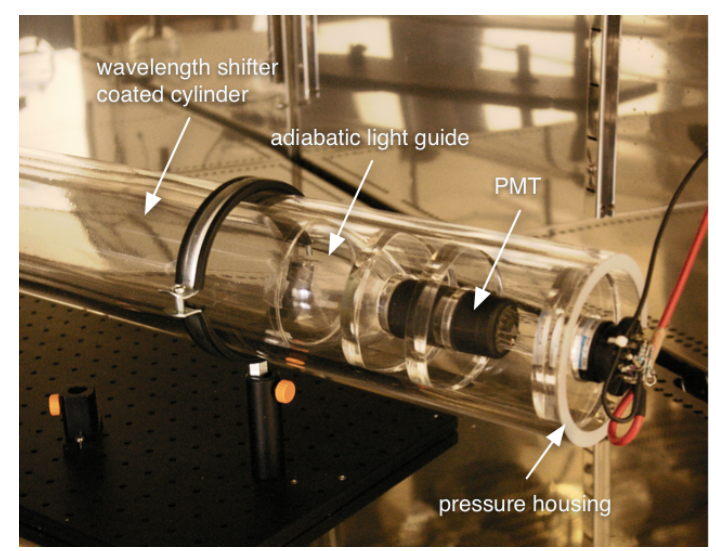

Figure 4: A initial attempt to assemble a prototype for noise measurements.

\subsection{Structuring the Inside of the Pressure Vessel}

As the light passes from ice to quartz glass to air, it goes through different refractive indices. As quartz glass has a greater refractive index than ice, nearly all the light passes through 
the first surface. Air on the contrary has a lower refractive index therefore the light with angles $\theta \geq 48.75^{\circ}=\arcsin \left(\frac{n_{\text {air }}=1}{n_{\text {ice }}=1.33}\right)$ does not enter the detector at the inner surface of the pressure vessel when the module is deployed in ice. This not only reduces the efficiency of the detector but also decreases the angular acceptance. In order to counteract both of these effects we investigate the use of hemispherical lenses to cover the inside of the pressure vessel. A first calculation indicates that this can give an efficiency gain of up to $37 \%$.

\subsection{Adiabatic Light Guide}
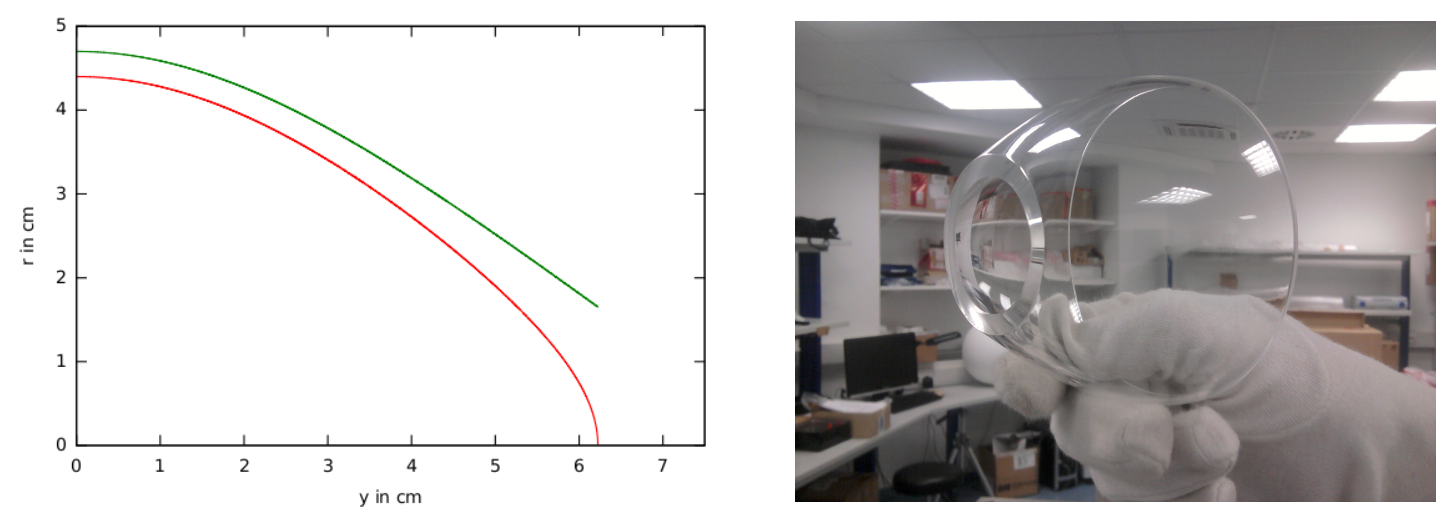

Figure 5: The calculated shape for an ideal adiabatic light guide with $r_{i, 0}=4.4 \mathrm{~cm}$ and $r_{0}=4.7 \mathrm{~cm}$ (left) and a first cast of this shape (right).

To redirect the light from the tube onto a PMT, an adiabatic light guide is used. To determine the optimal shape of the adiabatic light guide, we calculate the exterior radius $r$ and interior radius of the guide $r_{i}$ vs position on the symmetry axis $y$ with two methods. Both methods use a ray tracing simulation, one with cross section conservation and the second uses phase space conservation (Étendue). According to our calculations the outer surface can be approximated by a hyperbola as described by Eq. 5.1. $r_{0}$ is determined by the tubes radius, $\eta$ is the ellipticity that depends on the refractive index (currently determined by a fit to the simulation results). To calculate the shape of the inner surface, the fact of a constant cross section area as a consequence of Liouville's theorem can be used as described in eq. 5.2. In Eq. $5.2 r_{i, 0}$ is the initial inner radius determined by the wall thickness of the WLS tube. A plot of the functions is shown in Fig. 5 (left). Several prototypes with this shapes have been manufactured as shown in Fig. 5 (right) and will be tested

soon. $\quad r=r_{0}+\frac{r_{0}}{\eta^{2}-1}-\sqrt{\left(\frac{r_{0}}{\eta^{2}-1}\right)^{2}+\frac{r_{0}}{\eta^{2}-1} y^{2}}$

$$
r_{i}=\sqrt{r_{i, 0}^{2}+r^{2}-r_{0}^{2}}
$$

\subsection{Next Steps}

Current efforts are concentrating on improving the PMT-based setup for efficiency measurements and understanding the observed results. If the observed low value of $\varepsilon$ is indeed due to unaccounted in-efficiencies a gain in efficiency of $\approx 4$ in comparison to IceCube DOMs is in reach but even with $\varepsilon \approx 23 \%$ a factor of $\approx 2$ would be achieved. First prototypes can be deployed for testing with the deployment of the PINGU detector [5]. 


\section{Conclusion and Outlook}

The WOM is a low cost, low noise and high efficiency optical module. It is mainly developed to be used in IceCube-Gen2 and similar large volume neutrino Cherenkov detectors. However the concept is flexible and can be adapted for other types of detectors like in the surrounding background tagger of the SHiP experiment [3]. For the realization of this detector a high efficiency WLS paint has been developed. It shifts light from the range of $250-400 \mathrm{~nm}$ to $>400 \mathrm{~nm}$. First efficiency measurements with photodiodes showed capture and transport efficiencies in the range of $23 \%$ while first PMT measurements indicate that our measurements technique might use improvement and the value might be much higher. An adiabatic light guide has been produced that acts as an adapter between WLS tube and small PMTs. The noise rate of Hamamatsu R11920-100 PMTs has been investigated. Also the quartz glass pressure vessel in the necessary elongated shape has passed pressure testing. Currently we are close to assembling our first complete prototype to obtain final values on the detection parameters.

\section{References}

[1] IceCube-Gen2 Collaboration, "IceCube-Gen2: A Vision for the Future of Neutrino Astronomy in Antarctica", astro-ph.HE 2014, arXiv: 1412.5106

[2] S. Böser, M. Kowalski, L. Schulte, N. L. Strotjohann and M. Voge, Detecting extra-galactic supernova neutrinos in the Arctic ice, Astropart. Phys. 62 (2015) 54-65.

[3] SHiP Collaboration, A facility to Search for Hidden Particles (SHiP) at the CERN SPS, arXiv:1504.04956.

[4] Dustin Hebecker, Development of a Single Photon Detector with Wavelength-shifting and Light guiding Technology, Master Thesis, University of Bonn 2014

[5] The IceCube-PINGU Collaboration, "Letter of Intent: The Precision IceCube Next Generation Upgrade (PINGU)", January 2014, arXiv:1401.2046

[6] Eljen Technology, "EJ-298\#2 BLUE WAVESHIFTING PAINT", datasheet

[7] L. Schulte et. al., A large-area single photon sensor employing wavelength-shifting and light-guiding technology, ICRC 2013, arXiv:1307.6713S

[8] C. J. Bruegge et al., Use of Spectralon as a diffuse reflectance standard for in-flight calibration of earth-orbiting sensors, OPTICAL ENGINEERING April1993 Vol. 32 No.4 p. $805-814$

[9] T.Toyama et al. [CTA Consortium Collaboration], Novel Photo Multiplier Tubes for the Cherenkov Telescope Array Project, Proc. of 33rd ICRC, Rio de Janeiro, 2013.

[10] The IceCube Gen2 Collaboration, The IceCube Gen2 Digital Optical Module and Data Acquisition System, Proc. of 34rd ICRC, The Hauge, 2015 